\title{
Interleukin-2 Expression in Lupoid and Usual Types of Old World Cutaneous Leishmaniasis
}

\author{
Vahid Mashayekhi Goyonlo ${ }^{1,} ;$ Hesameldin Elnour $^{2} ;$ Klas Nordlind $^{2}$ \\ ${ }^{1}$ Department of Dermatology, Faculty of Medicine, Rsearch Center for Skin Disease and Cutaneous Leishmaniasis, Emam Reza Hospital, Mashhad University of Medical Sciences, \\ Mashhad, IR Iran \\ ${ }^{2}$ Department of Medicine, Unit of Dermatology and Venereology, Solna, Karolinska Instititutet, Stockholm, Sweden \\ ${ }^{*}$ Corresponding Author: Vahid Mashayekhi Goyonlo, Department of Dermatology, Faculty of Medicine, Rsearch Center for Skin Disease and Cutaneous Leishmaniasis, Emam Reza \\ Hospital, Mashhad University of Medical Sciences, Mashhad, IR Iran. Tel:+98-5118022490, E-mail: mashayekhiv@mums.ac.ir
}

Received: June 23, 2012; Revised: November 12, 2013; Accepted: October 6, 2014

\begin{abstract}
Background: Interleukin (IL)-2 plays a central role in T cell-dependent immune responses.
Objectives: We conducted this study to determine and compare IL-2 expression in lupoid and usual types of Old World Cutaneous Leishmaniasis (OWCL), using immunohistochemistry.

Patients and Methods: Thirteen paraffin-embedded specimens of lupoid and 12 specimens of usual types of OWCL were used. A mouse monoclonal anti IL-2 antibody was used for staining by the envision technique.

Results: There were strongly stained discrete foci of staining through inflammatory infiltrates of dermis and also in basal layers of epidermis and adnexal structures, with a distinctive pattern of hot spot activity foci (mean of $9.31 \pm 6.4$ versus $8.17 \pm 6.9$ foci per HPF for lupoid and usual types, respectively). The expression of IL-2 had no correlation with the pattern of granulomatous inflammation (tuberculoid, sarcoidal or mixed suppurative).

Conclusions: Interleukin-2 takes part in the immunological response of the granulomatous reaction of OWCL and is not statistically different between lupoid and usual types $(\mathrm{P}=0.674)$.
\end{abstract}

Keywords:Leishmaniasis; Interleukin-2; Granulomatous

\section{Background}

Lupoid Leishmaniasis is a clinically distinctive and immunologically unique form of old world cutaneous leishmaniasis (OWCL) that develops after $4 \%-10 \%$ of $L$. tropica-induced cutaneous leishmaniasis (CL) $(1,2)$. This chronic and non-healing form of OWCL is different from other chronic and non-healing forms of leishmaniasis in humans (muco-cutaneous and diffuse cutaneous types), because it is limited to site of inoculation and has a noninvasive behavior. Also immunologically, despite the presence of exaggerated hypersensitivity to leishmania $\mathrm{Ag}$, cellular immunity fails to resolve the lesion (1).

The role of immune response and immunoregulatory mediators in the clinical course of cutaneous leishmaniasis has been discussed and been the subject of many studies on the immunopathogenesis of CL $(3,4)$. Cytokines are the key element of immune response induction and development. The interleukins, named because their fundamental function appears to be communication between (inter-) various populations of white blood cells (leucocytes-leukin), are the largest group of cytokines. More than 25 years ago, interleukin (IL)-2 was identified as an autocrine secretory product required for long-term culture of helper (CD4+) T cells (5).

Early reports demonstrated that IL-2 promotes adaptive immunity by augmenting $\mathrm{T}$ cell proliferation, survival and effector Th1/Th2 differentiation $(6,7)$. More recently, it has been shown to promote expansion of regulatory $\mathrm{T}$ ( $\mathrm{T}$ reg) cells, a lineage that is critical for maintaining selftolerance (8). It is known that IL-2 secreting T cells are pluripotent, which means they are capable of differentiating into multiple lineages (Th1/Th2/T reg cells) (9). Although IL-2 is considered as a main mediator in immunopathogenesis of cutaneous leishmaniasis (3) yet to our knowledge there is so far no report of IL-2 immunohistochemical staining in granulomatous skin infiltrates including cutaneous leishmaniasis.

\section{Objectives}

This study aimed to demonstrate the presence of IL-2 in two different types of old world cutaneous leishmaniasis by immunohistochemistry, testing $\mathrm{ABC}$ and envision system techniques.

\section{Patients and Methods}

In a retrospective manner, paraffin-embedded specimens from fourteen cases of lupoid (chronic) leishmaniasis and 12 case of usual (acute) type of CL (from 
referrals between 2002 and 2004), were collected from the pathology archive of the leishmania clinic at Emamreza Hospital (Mashhad, Iran) and were included in this study. Cases of lupoid CL were included with the following criteria;

1)Clinically typical feature of chronic lupoid leishmaniasis

2) History of positive direct smear result for leishmania parasite at the beginning, before developing chronic course

3) Duration of more than two years

The cases of Usual CL were selected from the archive if they had:

1) Disease duration of less than 12 months (at most one year)

2) Presences of leishmania parasite in the histological examination

Paraffin embedded blocks were selected and after deparaffinization, sections (4 um) were dewaxed using XTRA SOLV (Savin Products, Randolph, MA, USA) for $2 \times$ 15 minute, and rehydrated in graded concentrations of ethanol. Hematoxylin and eosin ( $\mathrm{H}$ and $\mathrm{E}$ ) staining were done for each specimen. For immunohistochemical staining we performed antigen retrieval by heating in Tris-EDTA-buffer in a water bath for 25 minutes at $98^{\circ} \mathrm{C}$ and allowed cooling at room temperature. We used $0.3 \% \mathrm{H}_{2} \mathrm{O}_{2}$ for 15 minutes in a dark environment to block endogenous tissue enzyme activity. The next step was 40 minutes of incubation in $10 \%$ normal goat serum to minimize non-specific protein binding.

The primary antibody was a mouse monoclonal antihuman IL-2 IgG antibody obtained from MABTECH, Nacka Strand, Sweden; we incubated specimens at different dilutions (1/10-1/80) of the primary antibody for 60 minutes at room temperature. We also included blank and negative control sections during this step. Then the sections were washed in PBS, a peroxidase labeled polymer conjugated to goat-antimouse immunoglobulins (K4000, Envision, Dako) for 30 minutes or with the ABC (avidin-biotin complex horseradish peroxidase) technique, a biotinylated horse-antimouse antibody (BA2000; 1: 200; Vector Laboratories, Burlinghame, USA) were added and incubated overnight. The steps that followed included, avidin-biotin complex formation (PK6100, Vector Laboratories) for 30 minutes at room temperature, washing with PBS, chromogen incubation (amino-ethyl-carbazole (AEC)) (FK-4200, Vector Laboratories) for 15 minutes and counter staining with haematoxylin. The specimens were then mounted under glycerol gelatin. As blank and negative controls, the primary antibody and mouse IgG were used, respectively.

\section{Results}

Age, sex, duration of lesion and localization on the body are seen in Table 1. Hematoxylin and eosin stained sections showed granulomatous inflammatory infiltrate of tuberculoid type in 17 specimens (11 cases of lupoid and five cases of usual type) with well-formed foci of epithelioid cells containing giant cells of Langhans and foreign body type, surrounded by a prominent lymphocytic infiltration (Figure 1 -A). Seven cases of usual type had a diffuse mixed inflammatory infiltrate through the dermis including parasite loaded histiocyte, lymphocytes, some giant cells and few polymorphonuclear cells (Figure 1 -B). Two lupoid type cases showed very few lymphocytic infiltrates around granulomas resembling sarcoidal type granuloma and one case had lymphocyte predominant inflammatory infiltrate with a few small foci of epithelioid cells.

Immunohistochemical staining with the standard $\mathrm{ABC}$ technique was not conclusive and staining patterns were variable in different cases. Also it was not reproducible in even the same specimen. However, using the envision technique, we found a very characteristic and reproducible pattern of staining with an optimal dilution of 1/20 of primary antibody. There were discrete foci of strongly stained cells throughout the inflammatory infiltrate and some times in the epidermis and adnexal structures close to the basement membrane. Also there were positive cells between mononuclear inflammatory cell components of the granulomatous infiltrate (Figure 2). In the low power view of the microscope, they looked like foci of a very strong positive reaction. We called this pattern of foci of high IL-2 activity the "Hot spots" pattern. To compare IL-2 presentation in lupoid and usual types of OWCL, we counted the number of these hot spot foci through the slides. We used the Student's T test for data analysis.

The mean numbers of these foci of strongly stained IL-2 were variable from less than five per high power field (HPF) in both to at most 21 and 19 foci per HPF in lupoid and usual types, respectively. The mean for lupoid cases was $9.3 \pm 6.6$ and for usual type was $8.2 \pm 7.0$; there was no statistically significant difference between the two groups (P value $>0.5$ ).

\section{Discussion}

Interleukin-2 is a potent stimulator for macrophages and T cells. Its participation in immunopathogenesis of CL has been shown in many in vitro and experimental studies $(9,10)$ yet so far there is no report of IL-2 expression in granulomatous infiltrate of OWCL of human specimens. Here, we demonstrated the expression of IL-2 by inflammatory cells of granulomatous infiltrate in lupoid and usual types of OWCL with a uniform and reproducible pattern of Hot spot foci. Interleukin-2 expression had no statistically significant difference in the two groups yet regarding the obtained data its expression seems to be fundamental for initiation, provocation and continuance of inflammatory infiltration in both types of OWCL. 
Mashayekhi Goyonlo Vet al.

\begin{tabular}{|c|c|c|c|c|c|c|c|}
\hline \multicolumn{8}{|c|}{ Type of Cases } \\
\hline \multicolumn{4}{|c|}{ Lupoid Cases } & \multicolumn{4}{|c|}{ Usual (Acute) Cases } \\
\hline Case No. & Gender/Age, $y$ & Duration of Lesion, $y$ & Location & Case No. & Gender/Age, y & Duration of Lesion, mo & Location \\
\hline L 23 & $\mathrm{M} / 9$ & 5 & cheek & U 35 & $\mathrm{~F} / 63$ & 2 & hand \\
\hline L 26 & $\mathrm{~F} / 43$ & 11 & forearm & U 37 & $\mathrm{~F} / 50$ & 3 & forearm \\
\hline L 31 & $\mathrm{~F} / 48$ & 5 & nose & U 40 & $\mathrm{~F} / 68$ & 12 & arm \\
\hline L 33 & $\mathrm{M} / 12$ & 3 & cheek & $\mathrm{U} 41$ & $\mathrm{M} / 23$ & 8 & lip \\
\hline L 48 & $\mathrm{M} / 11$ & 5 & cheek & $\mathrm{U} 42$ & $\mathrm{M} / 21$ & 2.5 & forearm \\
\hline U 53 & $\mathrm{~F} / 41$ & 3 & cheek & $\mathrm{U} 43$ & $\mathrm{~F} / 60$ & 5 & hand \\
\hline L 59 & $\mathrm{M} / 12$ & 4 & forehead & U 46 & $\mathrm{~F} / 37$ & 7 & hand \\
\hline L 60 & $\mathrm{M} / 7$ & 5 & cheek & U 49 & $\mathrm{~F} / 73$ & 7 & forearm \\
\hline L 61 & $\mathrm{~F} / 42$ & 3 & cheek & U 57 & $\mathrm{M} / 51$ & 3 & forearm \\
\hline L 64 & $\mathrm{M} / 12$ & 5 & cheek & U 58 & $\mathrm{~F} / 51$ & 12 & forearm \\
\hline L 70 & $\mathrm{~F} / 14$ & 4 & cheek & U 68 & $\mathrm{~F} / 11$ & 7 & leg \\
\hline L 71 & $\mathrm{~F} / 13$ & 8 & cheek & U 69 & $\mathrm{~F} / 35$ & 4 & forearm \\
\hline L 75 & $\mathrm{~F} / 21$ & 6 & forehead & & & & \\
\hline
\end{tabular}

Figure 1. Hematoxylin and Eosin Staining
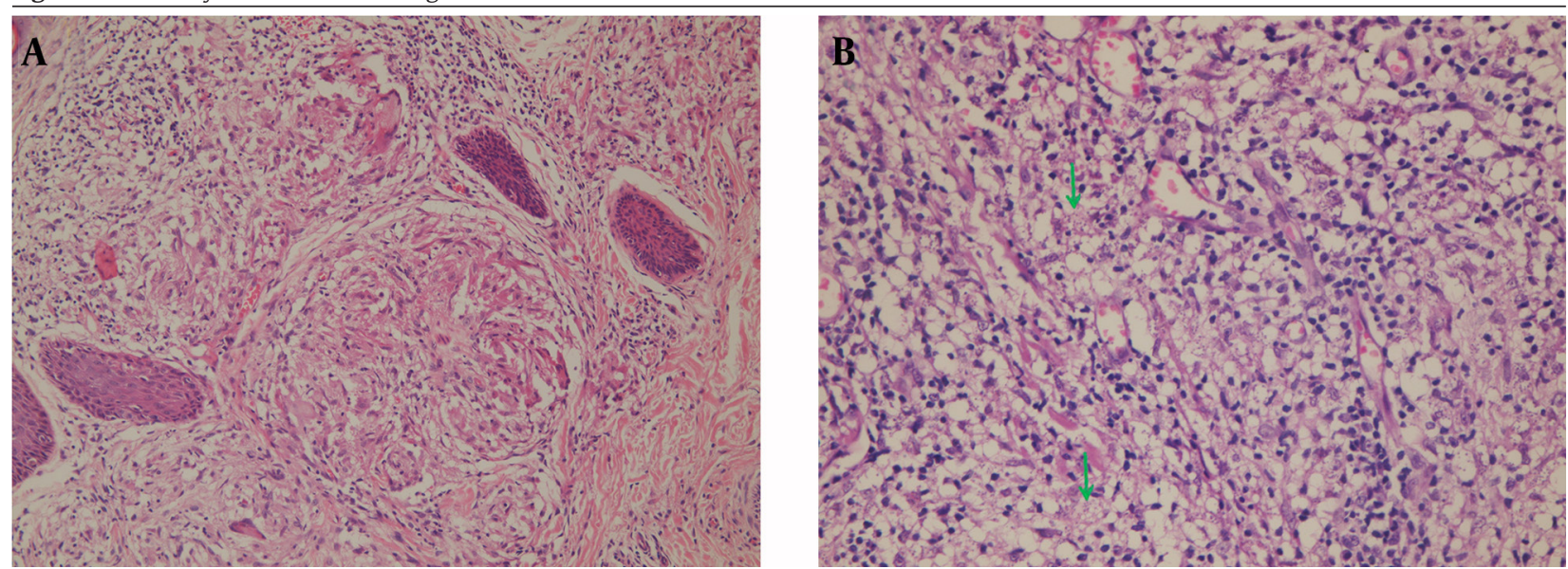

A) Well-formed foci of epithelioid and giant cells surrounded by a prominent lymphocytic infiltration in a lupoid case of cutaneous leishmaniasis (original magnification $\times 200$ ). B) Mixed inflammatory infiltrate through the dermis including parasite loaded histiocyte, lymphocytes, some giant cells and a few polymorphonuclear cells in a sample from a case with usual type of cutaneous leishmaniasis (original magnification $\times 400$ ).

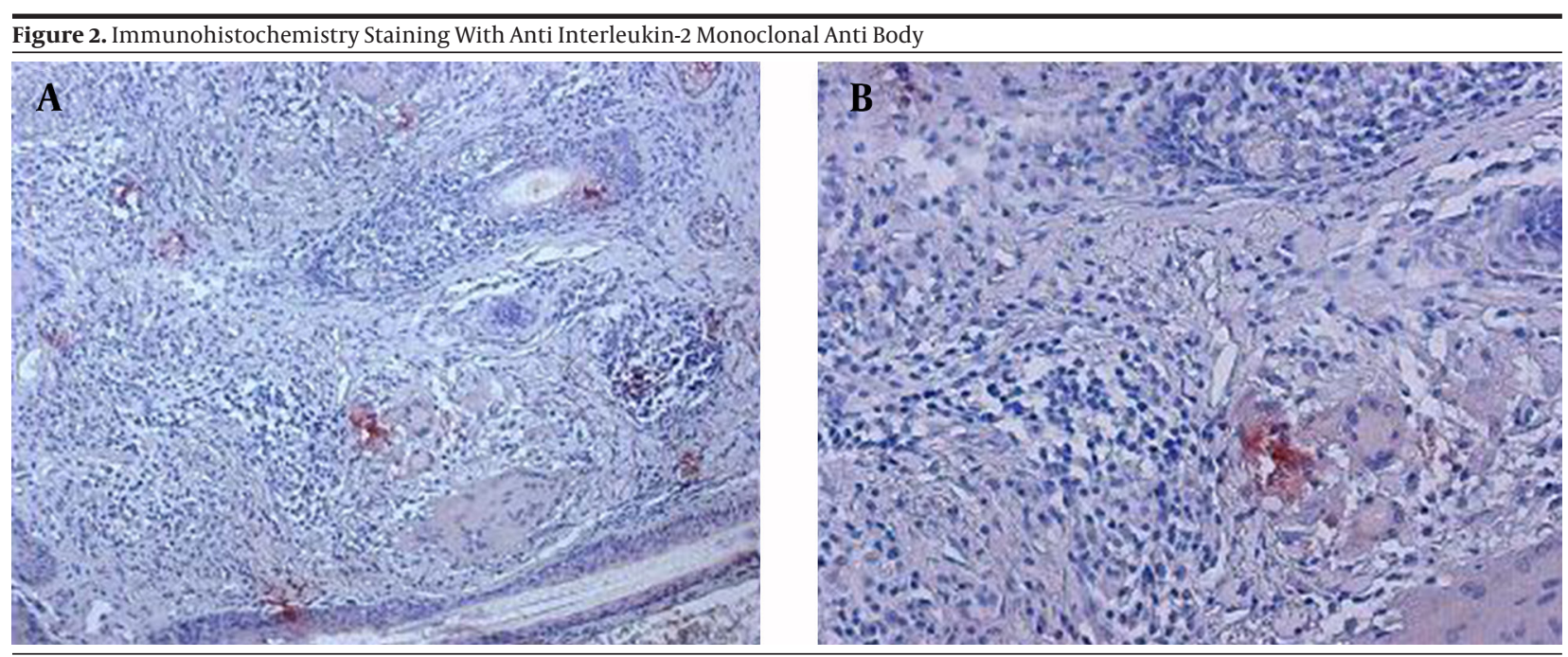

The image shows foci of strongly stained cells throughout the inflammation and epithelial structure (A; original magnifications: $\times 100$ and B; original magnifications $\times 200$ ). 
Interleukin-2 (IL-2) is a signaling molecule within the immune system that stimulates the growth of cytotoxic $\mathrm{T}$ cells and production of Interferon (IFN) by T helper cells, via binding to IL-2 receptors on lymphocytes. On the other hand, suppressor T cells exert IL-2 effects by adsorption or sequestering of IL-2. IFN- $\gamma$ is produced by different or the same cells that secrete IL-2 and in turn act on macrophages for induction of interleukin-1 (IL-1), which would participate in induction of IL-2. This IL-2-IFN- $\gamma$ cascade plays an important role in positive regulation of lymphocyte and macrophage function and maintains inflammatory reaction (11). Immunity in leishmaniasis is predominantly mediated by $\mathrm{T}$ lymphocytes and macrophages (3). The role of IL-2 in immunopathogenesis of leishmaniasis has been demonstrated in many studies. It activates macrophages for parasite digestion and triggers adaptive immunity by inducing T cells responses. In animal models during early infection with L. major, both resistant and susceptible hosts have been shown to exhibit mixed Th1/Th2 responses of CD4 + cell population with IL-2, IL-4 and IL-12 production. The IL- 4 and IL-12 production leads to Th2 and Th1 responses and are associated with susceptibility or resistance to leishmania infection, respectively (3). Studies on non-healing cases of CL in Iran have shown Th2 predominant T cell response to L. major Ag stimulation $(10,12)$. Other studies have demonstrated the role of IL-2 in IL-4 production in animal models of leishmania infection and anti-IL-2 or anti-IL-2 receptor antibodies have ameliorated the $L$. major infection $(3,13)$.

However, IL-2 participation in the lupoid type of old world cutaneous leishmaniasis is obscure. Recently Meymandi and coworkers demonstrated that Th1-like response is predominant in the acute active form and lupoid recidivans while Th2-like response is predominant in the chronic non-lupoid lesions. They used immunohistochemical staining for INF-gamma, TNF-alpha, IL-12 (markers for Th1 responses) and IL-4 (marker for Th2 responses) (14). Although this kind of classification for chronic non-healing forms of old world cutaneous leishmaniasis to lupoid recidivans and non-lupoid chronic subtypes is not clinically pervasive, their results are interesting and can be an initiative for more investigations on immunopathogenesis of this type of nonhealing CL that formally are considered as a type of Th2 predominant response to leishmania parasite $(10,12)$.

Regarding the pluripotential functions of IL-2 secreting T cells (9), we investigated its expression in inflammatory infiltrates of OWCL and did a comparison between acute (usual) and chronic (lupoid) forms. Our results show that IL-2 is expressed strongly in both types of lupoid and usual CL and although in the usual (acute) form expression is relative more prevalent than the lupoid type yet the difference is not statistically significant. Interleukin-2 has an important role in the positive regulation of lymphocyte and macrophage function and its expression in granulomatous infiltrate CL with interesting pattern of expression as foci of strongly positive cells, could be considered as the site of interaction between IL-2 producing and effector cells, the "Hot spot" of immune response induction and continuity.

Various methods have been used to determine the levels of expression of cytokines in tissues and culture media, such as bioassays, immunoassays and Northern blot analysis, to assess RNA levels, in situ hybridization (ISH) and immunohistochemical staining(IHC). The IHC methods permit the localization of cytokines within producing cells and within distinct compartments without the need to change the original microenvironment of the tissue $(15,16)$. To achieve optimal results, tissue processing, embedding and fixation are important for preservation of tissue antigens. Optimal dilutions for each anticytokine antibody should be used to avoid false-negative results or increased background staining. Signal amplification is dependent on the avidity of the binding between the primary and the secondary antibody (17). This study by using the envision technique obtained a conclusive and reproducible pattern of staining for IL-2 with 1:20 dilution of the primary antibody. The envision system amplified the target binding processes and was more competent to reveal IL-2 in our specimens.

Interleukin-2 is expressed in both lupoid and usual types of OWCL with a characteristic "hot spot" pattern in immunohistochemically-stained sections.

\section{Acknowledgements}

We thank Mrs Akram Momenzadeh for her assistance with the preparation of images included in this manuscript.

\section{Funding/Support}

This work was supported by the vice chancellor for research of Mashhad University of Medical Sciences, the Welander and Finsen foundation, and Karolinska Institute.

\section{References}

1. Lopez FV, Hay RJ. Parasitic worms and protozoa. In: Burns T, Breathnach S, Cox N, Griffiths C editors. Rooks text book of dermatology. 7 ed. Oxford: Blackwell science; 2004. pp. 32.1-32.48.

2. Sharifi I, Fekri AR, Aflatoonian MR, Khamesipour A, Mahboudi F, Dowlati Y, et al. Leishmaniasis recidivans among school children in Bam, South-east Iran, 1994-2006. Int J Dermatol. 2010;49(5):55761

3. Tripathi P, Singh V, Naik S. Immune response to leishmania: paradox rather than paradigm. FEMS Immunol Med Microbiol. 2007;51(2):229-42.

4. Hoseini SG, Javanmard SH, Zarkesh SH, Khamesipour A, Rafiei L, Karbalaie K, et al. Regulatory T-cell profile in early and late lesions of cutaneous leishmaniasis due to Leishmania major. J Res Med Sci. 2012;17(6):513-8.

5. Mier JW, Gallo RC. Purification and some characteristics of human T-cell growth factor from phytohemagglutinin-stimulated lymphocyte-conditioned media. Proc Natl Acad Sci U S A. 1980;77(10):6134-8.

6. Smith KA. Interleukin-2: inception, impact, and implications. Science. 1988;240(4856):1169-76. 
7. Waldmann TA, Dubois S, Tagaya Y. Contrasting roles of IL-2 and IL-15 in the life and death of lymphocytes: implications for immunotherapy. Immunity. 2001;14(2):105-10.

8. Malek TR, Bayer AL. Tolerance, not immunity, crucially depends on IL-2. Nat Rev Immunol. 2004;4(9):665-74.

9. Wang X, Mosmann T. In vivo priming of CD4 T cells that produce interleukin (IL)-2 but not IL-4 or interferon (IFN)-gamma, and can subsequently differentiate into IL-4- or IFN-gamma-secreting cells. J Exp Med. 2001;194(8):1069-80.

10. Ajdary S, Alimohammadian MH, Eslami MB, Kemp K, Kharazmi A. Comparison of the immune profile of nonhealing cutaneous Leishmaniasis patients with those with active lesions and those who have recovered from infection. Infect Immun. 2000;68(4):1760-4.

11. Pontzer $\mathrm{CH}$, Griggs ND, Johnson HM. Interleukin 2. Advances in Neuroimmunology. 1992;2(1):17-31.

12. Habibi GR, Khamesipour A, McMaster WR, Mahboudi F. Cytokine gene expression in healing and non-healing cases of cutaneous leishmaniasis in response to in vitro stimulation with recom- binant gp63 using semi-quantitative RT-PCR. Scand J Immunol. 2001;54(4):414-20.

13. Ben-Sasson SZ, Le Gros G, Conrad DH, Finkelman FD, Paul WE. IL-4 production by $\mathrm{T}$ cells from naive donors. IL-2 is required for IL-4 production. J Immunol. 1990;145(4):1127-36.

14. Meymandi S, Dabiri S, Shamsi-Meymandi M, Nikpour H, Kharazmi A. Immunophenotypic pattern and cytokine profiles of dry type cutaneous leishmaniasis. Arch Iran Med. 2009;12(4):371-6.

15. Robinson G, Ellis IA, MacLennan KA. Immunohistochemistry. In: Bancroft JD, Stevens A, Turner DR editors. Theory and Practice of Histological Techniques. 3 ed. New York: Churchill Livingstone; 1990. pp. 413-36.

16. Hoefakker S, Boersma WJ, Claassen E. Detection of human cytokines in situ using antibody and probe based methods. J Immunol Methods. 1995;185(2):149-75.

17. Huleihel M. Detection of cytokines by immunohistochemistry. In: O'Neill LAJ, Bowie A editors. Interleukin protocols.. Totowa: Humana Press Inc.; 2001. pp. 157-62. 